\title{
Association between cognitive impairment patient with solid cancer and insulin resistance
}

\author{
Kenji Gonda ${ }^{1,2,3,4^{*}}$, Kenji Yaginuma ${ }^{4}$, Yuichi Rokkaku${ }^{4}$, Shoichiro Horita' ${ }^{1}$ Yuko Maejima ${ }^{1}$ and Kenju Shimomura ${ }^{1}$
}

\begin{abstract}
Objectives: In an aging population, an increase in the number of elderly cancer patients with cognitive impairment is expected. The possible association between cancer and cognitive impairment is important to elucidate, because it can have a serious impact on quality of life. Here, we focused on glucose metabolism as a factor that links cancer and cognitive impairment.

Results: Thirteen subjects with solid cancers and cognitive impairment were recruited. As a control group, 14 subjects with cognitive impairment alone and 8 subjects with cancer alone were recruited. A Homeostatic Model Assessment of Insulin Resistance (HOMA-IR) and that of $\beta$-cell function (HOMA-B) were used. In comparison with patients with solid cancer alone, those with cognitive impairment alone and those with both cancer and cognitive impairment had increased HOMA-IR values. Insulin resistance was increased in patients with cognitive impairment alone and those with both cognitive impairment and solid cancer than in patients without cognitive impairment; however, $\beta$-cell function was not affected. The present data indicated that elderly cancer patients with high HOMA-IR score may be at a relatively high risk for developing cognitive impairment. Furthermore, early treatment to reduce insulin sensitivity may prevent cognitive impairment.
\end{abstract}

Keywords: Cognitive impairment, Solid cancer, HOMA-B, HOMA-IR

\section{Introduction}

An increase in the number of elderly patients with cancer and/or cognitive impairment is inevitable in an aging population. Cognitive impairment has recently been shown to affect up to $30 \%$ of patients with cancer [1-5] and can have a serious impact on the quality of life of both patients and families. However, the association between cognitive impairment and cancer remains unknown, and there has been no effective treatment for such patients. Recent studies have indicated that diabetes contributed to the development of cognitive impairment, such as Alzheimer's disease [6]. A number of reports have also indicated that hyperglycemia is a contributing factor to the progression of cancer [7]. Therefore, hyperglycemia or glucose intolerance may be the key

\footnotetext{
*Correspondence: gondake@fmu.ac.jp

${ }^{1}$ Department of Bioregulation and Pharmacological Medicine,

Fukushima Medical University School of Medicine, 1 Hikarigaoka, Fukushima 960-1295, Japan

Full list of author information is available at the end of the article
}

factor that links the development of cognitive impairment in patients with cancer [8]. Hyperglycemia can be induced by two different mechanisms; one is reduction of insulin secretion from pancreatic $\beta$-cells, and the other is increased insulin resistance in the target organs. The well-known cause of diabetes in a majority of cases in Asia is reduced insulin secretion; whereas that for the United States and Europe is insulin resistance. However, little is known on the contribution of hyperglycemia to cognitive impairment and cancer. Because the number of elderly cancer patients with cognitive impairment is expected to increase, understanding the underlying mechanism that links both diseases is important. In this study, we focused on the aspect that may link hyperglycemia with cognitive impairment and cancer. We applied a homeostasis model assessment (HOMA) to assess insulin resistance (HOMA-IR) and $\beta$-cell function (HOMA-B) in elderly patients with solid cancer (i.e., esophagus, gastric, colon, bile duct, prostate, breast, lung and ovary) and those with cognitive impairment, as well as in patients with both cancer and cognitive impairment. 


\section{Main text \\ Methods}

\section{Patient information}

A total of 13 subjects ( 7 men and 6 women) with an average age of 85 years and who had solid cancers and cognitive impairment were recruited (Table 1). For the control group, we recruited 14 subjects (6 men and 8 women) with an average age of 86 years and who had cognitive impairment alone and 8 subjects ( 5 men and 3 women) with an average age of 88 years and who had cancer alone. For the 8 patients with cancer alone, malignancy was based on tissue diagnosis.

\section{Research methods}

The Mini-Mental State Examination (MMSE) [9-11] and the Revised Hasegawa's Dementia Scale (HDS-R) tests were used for cognitive assessment of the patients [12].

Blood samples were collected at 07:00 a.m. after overnight fasting to measure fasting plasma glucose and fasting insulin levels.

\section{Statistical analysis}

The HOMA-IR and HOMA-B values were calculated using a HOMA calculator, which was available on the Diabetes Trials Unit website (http://www.dtu.ox.ac.uk). All the values were expressed as average + SD.

\section{Results}

\section{Comparison of $\beta$-cell function}

The HOMA-B values were not different among patients with cognitive impairment alone $(47.729 \% \pm 41.517 \%)$, cancer alone $(32.325 \% \pm 30.834 \%)$ and both (29.877\% $\pm 19.801 \%)$ (Fig. 1).

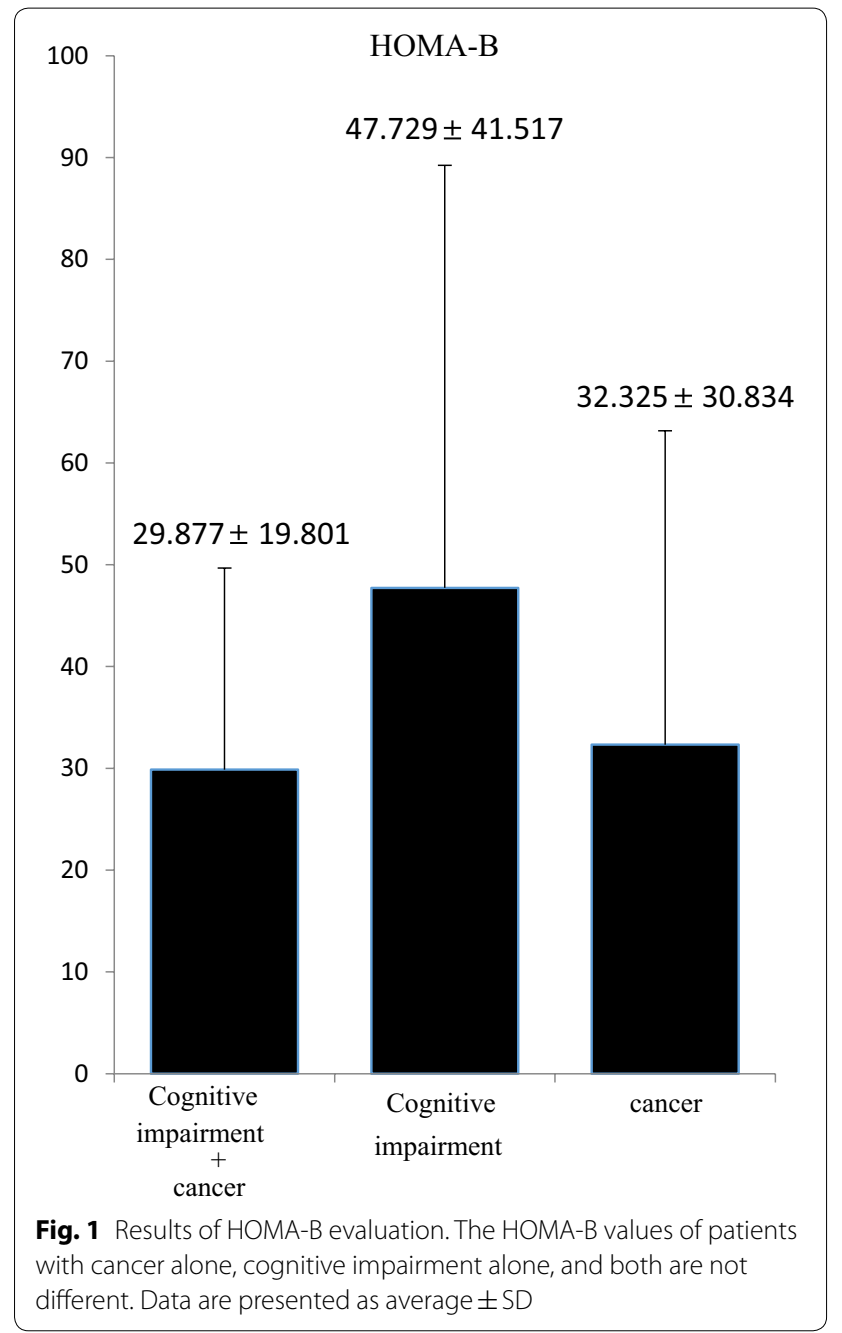

Table 1 The clinical and laboratory features of patients with both cognitive impairment and cancer

\begin{tabular}{|c|c|c|c|c|c|c|c|}
\hline Age & Gender & Cognitive impairment & Cancer & BS & IRI & IR & $\% \mathrm{~B}$ \\
\hline $80 s-90 s$ & $M$ & Alzheimer & Esophagus & 204 & 6.1 & 0.95 & 16.5 \\
\hline $80 s-90 s$ & $M$ & Alzheimer & Stomach & 192 & 11.6 & 1.77 & 30 \\
\hline $80 s-90 s$ & $\mathrm{~F}$ & Cerebrovascular & Stomach & 213 & 12.2 & 1.91 & 26 \\
\hline $70 s-80 s$ & $\mathrm{~F}$ & Cerebrovascular & Colon & 190 & 11.9 & 1.81 & 31.1 \\
\hline $90 s-100 s$ & M & Cerebrovascular & Colon & 273 & 10.4 & 1.91 & 15.1 \\
\hline $80 s-90 s$ & M & Alzheimer & Colon & 195 & 8.5 & 1.31 & 23 \\
\hline $80 s-90 s$ & $F$ & Alzheimer & Pancreas & 318 & 10.3 & 2.38 & 11.8 \\
\hline $80 s-90 s$ & $M$ & Alzheimer & Bile duct & 191 & 11.2 & 1.71 & 29.4 \\
\hline $90 s-100 s$ & $M$ & Cerebrovascular & Prostate & 220 & 13.3 & 2.1 & 26.3 \\
\hline $70 s-80 s$ & $\mathrm{~F}$ & Alzheimer & Breast & 110 & 12.7 & 1.72 & 90.4 \\
\hline $90 s-100 s$ & $M$ & Alzheimer & Lung & 198 & 16.3 & 2.48 & 37 \\
\hline $80 s-90 s$ & $\mathrm{~F}$ & Parkinson & Ovary & 200 & 15.4 & 2.35 & 34.8 \\
\hline $70 s-80 s$ & $\mathrm{~F}$ & Alzheimer & Vulvar & 275 & 12.2 & 2.25 & 17 \\
\hline
\end{tabular}

BS blood sugar (mg/dL), IRI insulin ( $\mu \mathrm{U} / \mathrm{mL})$, IR Homeostatic Model Assessment of Insulin Resistance (HOMA-IR), \%B Homeostatic Model Assessment of $\beta$-cell Function (HOMA-B) (\%) 


\section{Comparison of insulin resistance}

Insulin resistance was different among the groups. In particular, the HOMA-IR values were higher in patients with cognitive impairment alone $(1.307 \pm 0.673)$ and in those with both cognitive impairment and cancer $(1.896 \pm 0.435)$ than in those with cancer alone $(0.645 \pm 0.196)$ (Fig. 2).

\section{Clinical features of the patients and the relationship between blood sugar (BS) and immune-reactive insulin (IRI) level}

Compared with patients with cognitive impairment alone, those with both cognitive impairment and cancer had higher levels of BS $(213.769 \pm 51.134$ vs. $160.429 \pm 51.831 \mathrm{mg} / \mathrm{dL})$ and IRI $(11.700 \pm 2.653$ vs. $8.993 \pm 4.739 \mu \mathrm{U} / \mathrm{mL}$ ) (Table 1$)$.

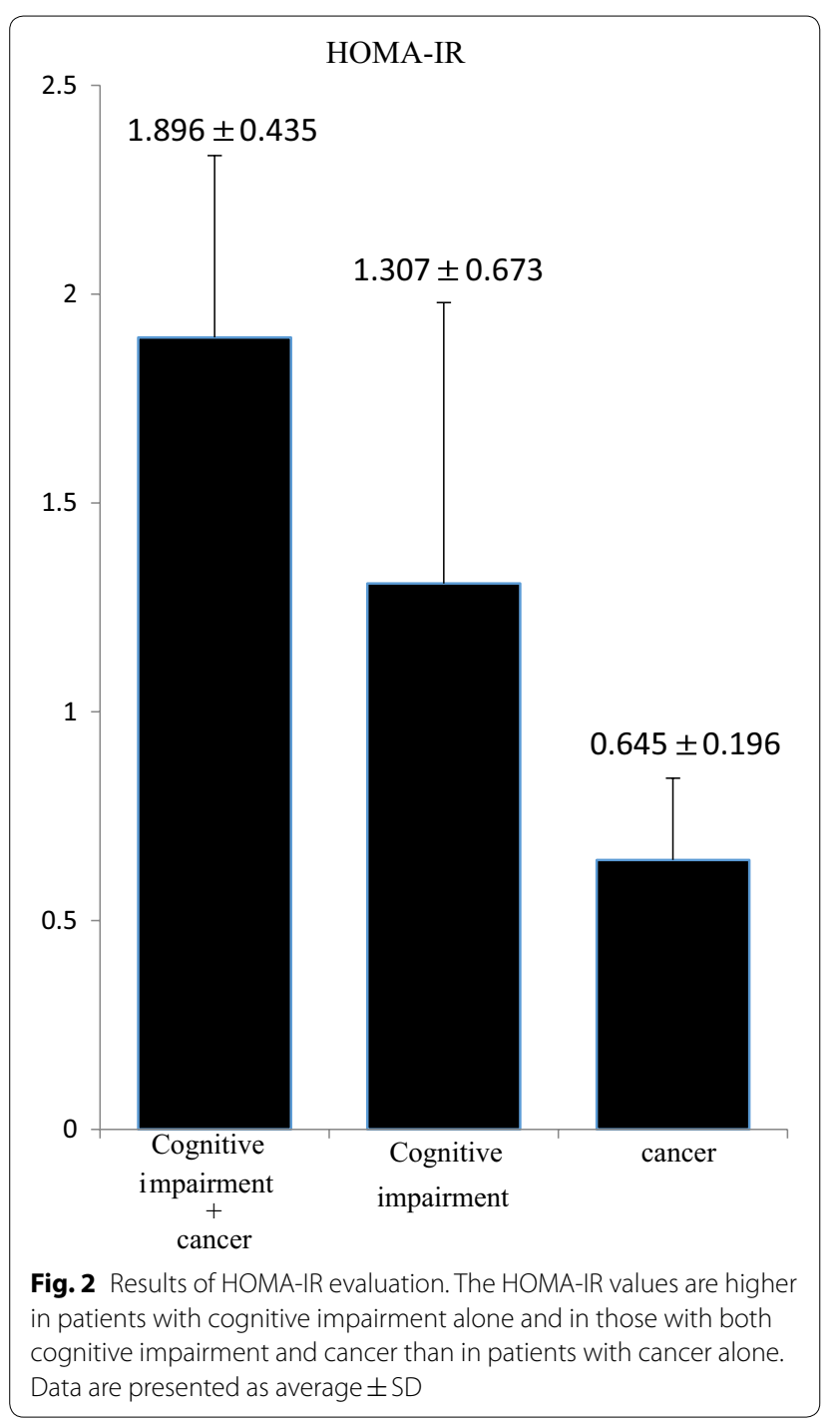

\section{Discussion}

In the present study, we have shown that insulin resistance, based on the HOMA-IR, was increased in patients with cognitive impairment, regardless of the presence of solid cancer, compared with that in cancer patients without cognitive impairment. The similar HOMA-IR values between patients with cognitive impairment alone and those with both cognitive impairment and cancer suggested that the presence of solid cancer itself did not contribute to the development of insulin resistance in cancer patients.

To date, many studies have indicated the relationship between insulin resistance and cancer development or progression [13]. However, in the present study, the HOMA-IR was significantly lower in patients with cancer alone than in those with cognitive impairment alone. Therefore, the contribution of insulin resistance to cancer development and progression was not evident, and further studies are required to validate these findings. On the other hand, recent epidemiologic and basic scientific investigations have suggested an association and common pathologic mechanisms between hyperglycemia and cognitive impairment, including Alzheimer's disease [7]. Interference in the insulin signal processing in the brain has been indicated as the mechanism for the development of cognitive impairment in diabetic patients. Wan et al. reported that insulin induced functional postsynaptic gamma-aminobutyric acid (GABA) receptors in the brain [14]. Furthermore, low insulin sensitivity was reported to contribute to decreased acetylcholine synthesis, which leads to Alzheimer's disease [15]. Our present data suggested that cancer patients are not exempted from developing hyperglycemia due to low insulin sensitivity, which induces cognitive impairment. However, based on the similar HOMA-IR values between patients with cognitive impairment alone and those with both cancer and cognitive impairment, insulin resistance may not be the sole contributing factor to the development of cognitive impairment in patients with solid cancers. Interestingly, a majority of Japanese diabetic patients have been known to have insulin secretion deficiency but not insulin resistance [16]. Our present data implied the importance of HOMA-IR measurement in elderly cancer patients, because those with high HOMA-IR scores may be at a high risk for developing cognitive impairment and may benefit from early treatment, such as the use of biguanide, to reduce insulin sensitivity. However, further studies are required to investigate the effects of biguanide on the development of cognitive impairment in elderly cancer patients.

In summary, our results suggested that insulin resistance but not $\beta$-cell function was increased in patients with cognitive impairment alone and those with both 
cognitive impairment and solid cancer than in cancer patients without cognitive impairment. Elderly cancer patients with insulin resistance may be at a high risk for developing cognitive impairment, which may be prevented by early treatment that reduces insulin sensitivity.

\section{Limitations}

Due to the small number of subjects, this study cannot show a cause-effect relationship strictly. Social acceptance and recall bias were also possible confounding factors.

\section{Abbreviations \\ HOMA-IR: homeostasis model assessment was used to assess insulin resist- ance; HOMA-B: homeostasis model assessment was used to assess $\beta$-cell function; BS: blood sugar; IRI: immune-reactive insulin.}

\section{Acknowledgements}

The authors would like to thank the staff, study participants, data collectors, and supervisors of Fukushima Medical University and the Japan Community Healthcare Organization Nihonmatsu Hospital, Specialized Hospital.

\section{Authors' contributions}

$K G, K Y$, and $Y R$ performed patient recruitment and clinical investigation. $K G$ $\mathrm{KY}, \mathrm{YR}, \mathrm{SH}, \mathrm{YM}$, and KS conceived the study, participated in its design, and coordinated and helped draft the manuscript. All authors read and approved the final manuscript.

\section{Funding}

No funding was obtained for this research work.

\section{Availability of data and materials}

The dataset in the current study is available from the corresponding author upon request.

\section{Ethics approval and consent to participate}

The institutional review board and the local ethics committee (the Ethics Review Board of Japan Community Healthcare Organization Nihonmatsu Hospital, no. 00037) approved the study. All procedures were performed in accordance with the ethical standards of the responsible committee on human experimentation at Fukushima Medical University and with the Helsinki Declaration of 1975, as revised in 2000. Each author certifies that all investigations were conducted in conformity with the ethical principles.The average MMSE score of the 13 subjects with solid cancers and cognitive impairment was 22.6, and that for the 14 subjects with cognitive impairment alone was 21.4. Based on the $\leq 23$ MMSE score of the patients in this study, it may be said that the patients were in cognitive decline. We reviewed the consent form that was submitted to the ethics review board, in reference to the background and design of the LASA (The Longitudinal Aging Study Amsterdam) study. We obtained the qualifications for specialist in intractable disease designation from the governor of Fukushima. An example of an intractable disease designated by the Japanese Ministry of Health, Labour and Welfare is frontal head dementia or dementia in young people. Written informed consent to participate in the study was obtained from the legal guardians or representatives of the 13 subjects with solid cancers and cognitive impairment and the 14 subjects with cognitive impairment alone.

\section{Consent for publication}

Not applicable.

\section{Competing interests}

The authors declare that they have no competing interests.
Japan. ${ }^{2}$ Daido Obesity and Metabolism Research Center, 123 Daido, Naha, Okinawa 902-0066, Japan. ${ }^{3}$ Center for Medical Genetics and Immunology, Fukushima Medical University School of Medicine, 1 Hikarigaoka, Fukushima 960-1295, Japan. ${ }^{4}$ Japan Community Healthcare Organization Nihonmatsu Hospital, 1-553 Narita, Nihonmatsu, Fukushima 964-8501, Japan.

Received: 18 August 2019 Accepted: 16 October 2019

Published online: 28 October 2019

\section{References}

1. Denlinger CS, Ligibel JA, Are M, Baker KS, Demark-Wahnefried W, Friedman DL, et al. Survivorship: cognitive function, version 1.2014. J Natl Compr Cancer Netw. 2014;12(7):976-86.

2. Janelsins MC, Kesler SR, Ahles TA, Morrow GR. Prevalence, mechanisms, and management of cancer-related cognitive impairment. Int Rev Psychiatry. 2014;26(1):102-13.

3. Jean-Pierre P, Winters PC, Ahles TA, Antoni M, Armstrong FD, Penedo $F$, et al. Prevalence of self-reported memory problems in adult cancer survivors: a national cross-sectional study. J Oncol Pract. 2012;8(1):30-4.

4. Moore HC. An overview of chemotherapy-related cognitive dysfunction, or'chemobrain'. Oncology. 2014;28(9):797-804.

5. Wefel JS, Vardy J, Ahles T, Schagen SB. International Cognition and Cancer Task Force recommendations to harmonise studies of cognitive function in patients with cancer. Lancet Oncol. 2011;12(7):703-8.

6. Janson J, Laedtke T, Parisi JE, O'Brien P, Petersen RC, Butler PC. Increased risk of type 2 diabetes in Alzheimer disease. Diabetes. 2004;53(2):474-81.

7. Akter K, Lanza EA, Martin SA, Myronyuk N, Rua M, Raffa RB. Diabetes mellitus and Alzheimer's disease: shared pathology and treatment? $\mathrm{Br} J \mathrm{Clin}$ Pharmacol. 2011;71(3):365-76.

8. Zheng F, Yan L, Yang Z, Zhong B, Xie W. HbA1C, diabetes and cognitive decline: the English Longitudinal Study of Ageing. Diabetologia. 2018;61(4):839-48.

9. Folstein MF, Folstein SE, McHugh PR. "Mini-mental state". A practical method for grading the cognitive state of patients for the clinician. J Psychiatr Res. 1975;12(3):189-98.

10. Tsoi KK, Chan JY, Hirai HW, Wong SY, Kwok TC. Cognitive tests to detect dementia: a systematic review and meta-analysis. JAMA Intern Med. 2015;175(9):1450-8.

11. Velayudhan L, Ryu SH, Raczek M, Philpot M, Lindesay J, Critchfield M, et al. Review of brief cognitive tests for patients with suspected dementia. Int Psychogeriatr. 2014;26(8):1247-62.

12. Jeong JW, Kim KW, Lee DY, Lee SB, Park JH, Choi EA, et al. A normative study of the Revised Hasegawa Dementia Scale: comparison of demographic influences between the Revised Hasegawa Dementia Scale and the Mini-Mental Status Examination. Dement Geriatr Cogn Disord. 2007;24(4):288-93.

13. Arcidiacono B, liritano S, Nocera A, Possidente K, Nevolo MT, Ventura V, et al. Insulin resistance and cancer risk: an overview of the pathogenetic mechanisms. Exp Diabetes Res. 2012;2012:789174.

14. Wan Q, Xiong ZG, Man HY, Ackerley CA, Braunton J, Lu WY, et al. Recruitment of functional GABA(A) receptors to postsynaptic domains by insulin. Nature. 1997;388(6643):686-90.

15. Rivera EJ, Goldin A, Fulmer N, Tavares R, Wands JR, de la Monte SM. Insulin and insulin-like growth factor expression and function deteriorate with progression of Alzheimer's disease: link to brain reductions in acetylcholine. J Alzheimers Dis. 2005;8(3):247-68.

16. Aoyama-Sasabe S, Fukushima M, Xin X, Taniguchi A, Nakai Y, Mitsui R, et al. Insulin secretory defect and insulin resistance in isolated impaired fasting glucose and isolated impaired glucose tolerance. J Diabetes Res. 2016;2016:1298601.

\section{Publisher's Note}

Springer Nature remains neutral with regard to jurisdictional claims in published maps and institutional affiliations.

\footnotetext{
Author details

1 Department of Bioregulation and Pharmacological Medicine, Fukushima Medical University School of Medicine, 1 Hikarigaoka, Fukushima 960-1295,
} 